PharmacoEconomics \& Outcomes News 885, p4 - 21 Aug 2021

\title{
Costs of providing COVID 19-protective measures during routine immunisation in lower-income countries?
}

In order to minimise COVID-19 transmission and ensure the safety of health workers and the community, the startup costs of providing COVID-19 protection during immunisation for preventable diseases in low- to middle-income countries would be around \$US466-\$799 for routine fixed-site delivery and $\$ 12-\$ 220$ for routine outreach immunisation strategies.

The study, published in Vaccine, modelled the additional resource requirements and costs (2020 US dollars) associated with modifying supplementary immunisation campaigns and routine immunisation services via fixed sites and outreach to minimise COVID-19 transmission; a real-world case study of Tanzania was also conducted. Four cost categories were considered: (1) personal protective equipment and infection prevention and control measures; (2) physical distancing and screening during immunisation; (3) delivery strategy changes (changes in immunisation session sizes and frequency); and (4) other operational cost increases (additional social mobilisation, training, and hazard pay for health workers).

The additional costs per facility of implementing COVID-19 protective measures could range from $\$ 466-\$ 799$ for routine fixed-site delivery, $\$ 12-\$ 220$ for routine outreach delivery, and \$12-\$108 per immunisation campaign site. Recurrent monthly costs could be \$137-\$1024 for fixed-site delivery and \$152-\$848 for outreach delivery per facility, and a \$0.32-\$0.85 increase in the cost per dose during immunisation campaigns. Using real-world data from Tanzania, the estimated cost increase was $89 \%-147 \%$ based on an annual programme cost of \$138 million, "but we might expect cost increases to vary according to the annual programme cost" in other country scenarios, point out the authors.

Banks C, et al. Cost of vaccine delivery strategies in low- and middle-income countries during the COVID-19 pandemic. Vaccine : 29 Jun 2021 . Available from: URL: http:// doi.org/10.1016/j.vaccine.2021.06.076 